\title{
Pancreatitis aguda
}

Martín Adrián Bolívar-Rodríguez*, Marcel Antonio Cázarez-Aguilar, Carlos Arturo Respardo-Ramírez, Rodolfo Fierro-López, Diana Tello-García.

Departamento de Cirugía General, Centro de Investigación y Docencia en Ciencias de la Salud, Universidad Autónoma de Sinaloa, Culiacán, Sinaloa, México

DOI http://dx.doi.org/10.28960/revmeduas.2007-8013.v8.n3.005

Recibido 12 Febrero 2018, aceptado 28 Mayo 2018

RESUMEN

La pancreatitis aguda es una enfermedad que se presenta como abdomen agudo y es una causa importante de ingreso hospitalario; su incidencia ha mostrado un incremento probablemente por aumento de los factores de riesgo como la obesidad. Su presentación clínica es muy variable, desde una enfermedad leve con recuperación en pocos días con tratamiento no complejo, hasta enfermedad grave y fatal que no responde a ningún tratamiento, siendo una enfermedad que se caracteriza por tener una evolución que no se puede predecir y que actualmente esta totalmente establecido que se relaciona más estrechamente con el desarrollo de falla orgánica múltiple que por la necrosis pancreática, con una morbimortalidad importante, requiriendo de un diagnóstico precoz y manejo oportuno para evitar complicaciones y muertes.

PALABRAS CLAVE: Pancreatitis aguda, diagnóstico, tratamiento.

\section{ABSTRACT}

Acute pancreatitis is a disease that presents as acute abdomen and is an important cause of hospital admission; its incidence has shown an increase probably due to an increase in risk factors such as obesity. Its clinical presentation is very variable, from a mild illness with recovery in a few days with non-complex treatment, to severe and fatal disease that does not respond to any treatment, being a disease characterized by having an evolution that can not be predicted and that currently, it is fully established that it is more closely related to the development of multiple organ failure than to pancreatic necrosis, with significant morbidity and mortality, requiring early diagnosis and timely management to avoid complications and deaths.

KEY WORDS: Acute pancreatitis, diagnosis, treatment.

\section{Introducción}

Pancreatitis aguda (PA) se define como la inflamación del páncreas debido a la inadecuada activación enzimática, con elevacion de las enzimas pancreáticas hasta tres veces de su límite superior normal, con afectación local y sistémica, que puede llevar a falla orgánica múltiple. La colelitiasis y el alcohol son las dos principales causas, siendo hasta $50-70 \%$ de origen biliar. ${ }^{1}$

El páncreas fue descubierto por Herophilus, un anatomista y cirujano griego en Chalcedo (ahora Kadikoy; Istanbul) en $336 \mathrm{AC}^{2}$

Durante la historia del páncreas sobresale Galeno de Pérgamo Romano (129-201/216 A.C.), quien

\footnotetext{
*Correspondencia: Dr. Martín Adrián Bolívar Rodríguez, Eustaquio Buelna no. 91, colonia Gabriel Leyva, cp. 80030. Culiacán, Sinaloa, México.Tel.: 667-713-26-06

Celular: 667-996-87-56. Correo: bolivarmartin64@hotmail.com
}

habló sobre la cirrosis pancreática; Giovanni Battista Morgagni (1682-1771) en 1761 describió por primera vez el pseudoquiste pancreático en un cadáver; Johann Ritter von Oppolzer (1808-1871), en 1861 diagnosticó la necrosis pancreática en un paciente vivo ${ }^{1,3}$ y en 1886 Nicholas Senn (18441908) realizó la primera necrosectomía en un paciente con PA severa. ${ }^{4}$

El primer intento predictivo para PA fue introducido por John H. C. Ranson (1938-1995) en 1974, la escala que lleva su nombre. Hans Günther Beger ${ }^{5}$ fue el primer cirujano que realizó una necrosectomía pancreática programada, procedimiento que era llevado a cabo tempranamente en el curso de PA severa.

Lloyd E. Ratner ${ }^{5}$ en 1992 reportó que el 40\% de los pacientes operados con necrosis pancreática 
inicialmente estéril desarrollaban infección secundaria durante el periodo postoperatorio.

En 1992 en Atlanta se realizó la primera clasificación sobre pancreatitis, estandarizándola clínica, laboratorial y radiológicamente. ${ }^{6}$

\section{Epidemiología}

La PA es la causa gastrointestinal más común de admisión hospitalaria en Estados Unidos de América. ${ }^{1}$ Se asocia con una elevada morbimortalidad y costos hospitalarios. Su incidencia ha ido en aumento de 5 hasta $30 \%$ en los casos más severos, sin embargo, se reporta un promedio de entre 4.9 y 73.4 casos por cada 100,000 habitantes. ${ }^{3}$ Se ha atribuído un aumento en la incidencia debido a los estudios diagnósticos, como la Tomografía computarizada (TC) y el Ultrasonido (US) endoscópico.

La nueva clasificación de Atlanta divide la pancreatitis en 3 tipos: leve; moderadamente severa y severa. ${ }^{7}$ El $80-85 \%$ son PA leve y $15-20 \%$ PA severas con complicaciones locales y/o sistémicas. $^{8}$

La PA biliar es más frecuente en las mujeres; sin embargo, el riesgo de desarrollar PA severa es mayor en los hombres. ${ }^{9}$

\section{Etiología}

Existen múltiples factores que pueden desencadenar una PA, las etiologías más frecuentes son la biliar y la alcohólica. La litisis biliar representa un $50-70 \%$ de los casos, ${ }^{1}$ aproximadamente el $7 \%$ de los pacientes con litiasis vesicular desarrollará pancreatitis a lo largo de su vida siendo los litos menores a $5 \mathrm{~mm}$ los que se asocian más a PA. ${ }^{3}$

El consumo de alcohol es la segunda causa de PA en un $5 \%$ de los casos, en pacientes con un consumo mínimo de $50 \mathrm{~g} /$ día por al menos 5 años. La tercera causa en frecuencia es la hipertrigliceridemia que representa hasta el $4 \%$ de los cuadros agudos. Una concentración de 1,000 mg/dl es desencadenante del cuadro. ${ }^{3}$

Las siguientes causas son por medicamentos, autoinmune, alteraciones pancreáticas como el páncreas divisum, y en un porcentaje de entre un 10$30 \%$ no se llega a identificar la causa, se conocen como PA idiopática, aunque se cree que muchas de estas realmente tienen un origen biliar, por microlitiasis. $^{9}$

\section{Fisiopatología}

En términos de fisiopatología la pancreatitis aguda se divide en temparna y tardía. La fase temprana ocurre dentro de la primera semana, mientras que la fase tardía comienza a partir de la segunda semana y puede durar varios meses. ${ }^{6,7}$ Las teorías más estudiadas por la cual la etiología biliar causa pancreatitis son: el reflujo biliar hacia los conductos pancreáticos por la obstrucción distal de la vía biliar, la hipertensión retrógrada en los conductos pancreáticos y la entrada de bilis al sistema pancreático ocasionando activación de tripsina y lesión tisular. ${ }^{3}$

En la PA se presenta una activación intrapancreática de proenzimas (zimógenos) produciendo lisis y autodigestión pancreática. Algunos agentes como la bilis inhiben la exocitosis de los gránulos 
de zimógenos, generando un acúmulo intracitoplamástico favoreciendo la fusión con los lisosomas, llevando a una activación de enzimas en cascada y un fenómeno de retroalimentación por lesión-activación. ${ }^{9}$

El tripsinógeno es el responsable de todas las complicaciones sistémicas, se activa la elastasa produciendo lesión vascular, favoreciendo la hemorragia; la calicreína produce dolor y aumenta la permeabilidad vascular, favoreciendo el shock. La fosfolipasa activada favorece el paso de lecitina a lisolecitina, que a su vez es capaz de escindir los fosfolípidos generando necrosis por coagulación. 2,9

En la mayoría de los casos el páncreas presenta únicamente edema, el cual revierte y se recupera en una semana aproximádamente. Sólo $20 \%$ de los casos serán graves, presentando complicaciones locales o sistémicas, causando un desbalance proteasas-antiproteasas con activación del complemento y del sistema de las quininas. La liberación de citocinas proinflamatorias, como el factor de necrosis tumoral alfa, y las interleucinas 1, 6 y 8; metabolitos del ácido araquidónico como prostaglandinas y leucotrienos, incrementan las lesiones microcirculatorias y aumentan la permeabilidad vascular, lo que induce hemorragia, trombosis y necrosis pancreática. ${ }^{9}$ Como parte de su fisiopatología la hipercoagulabilidad con microtrombosis, daño endotelial y salida de radicales libres, aumenta la permeabilidad capilar. ${ }^{7}$
En pacientes obesos las complicaciones son mayores ya que la inflamación del tejido adiposo produce mediadores que contribuyen a la respuesta inflamatoria sistémica, el tejido adiposo peripancreático ha demostrado ser un factor aún más importante que el Indice de Masa Corporal o la circunferencia de la cintura. ${ }^{2}$

\section{Diagnóstico}

El diagnóstico de PA se realiza con al menos dos de los siguientes puntos: clínica típica de pancreatitis, lipasa y amilasa sérica 3 veces por arriba de su límite superior normal y características imagenológicas de pancreatitis aguda, por ultrasonido, tomografía y/o resonancia magnética.

El síntoma más común es el dolor abdominal y se debe a la dilatación de la cápsula pancreática debido al edema y es referido por el paciente como agudo, progresivo, constante y de intensidad moderado a muy intenso, localizado en todo el hemiabdomen superior en la profundidad del epigastrio, en el $50 \%$ de los pacientes se produce una irradiación «en cinturón» hacia la espalda. Las náuseas y vómitos se presentan en el $80-90 \%$ de los pacientes, así como distensión abdominal por íleo. ${ }^{9}$

En raras ocasiones se observa equimosis en flancos (signo de Grey Turner) y periumbilical (signo de Cullen) causado por extravasación hemorrágica y nos hablan de PA severa. ${ }^{10}$

La determinación de y-glutamiltransferasa (y-GT), fosfatasa alcalina y bilirrubina como parámetros de colestasis, así como las transaminasas alanina 
aminotransferasa (ALAT) y aspartato aminotransferasa (ASAT) pueden indicar una génesis biliar de pancreatitis, un aumento de ALAT a más de 3 veces su valor normal dentro de las 48 horas del inicio de los síntomas indica pancreatitis biliar (valor predictivo positivo mayor del 85\%). ${ }^{10}$

El diagnóstico de PA con una tira reactiva de tripsinógeno-2 en orina es muy eficaz, con una capacidad diagnóstica equivalente a las enzimas pancreáticas séricas. ${ }^{11}$

EI US debe realizarse a todos los pacientes en los que se sospeche colelitiasis o coledocolitiasis. La colangioresonancia magnética se recomienda en los pacientes con elevación enzimática y en los cuales la vía biliar no se ve adecuadamente en el US. ${ }^{12}$

La TC dinámica se indica para diferenciar entre pancreatitis intersticial (90-95\% de los casos) de la necrosante y/o cuando se sospecha de complicaciones locales (48-72 hrs), salvo que esté contraindicado (enfermedad renal), se debe administrar contraste endovenoso para evaluar la necrosis pancreática, iniciando el estudio 40 segundos después de la inyección. ${ }^{12,13}$ Cuando se realiza la TC en su fase arterial en estadío temprano la mala definición del páncreas puede deberse a isquemia reversible más al encontrase posterior a la segunda semana nos habla de una necrosis pancreática $^{11}$ y es muy efectiva para guiar el tratamiento de posibles complicaciones. ${ }^{14}$

\section{Severidad y pronóstico}

La PA según el Consenso de Atlanta 2012 se clasifica en leve, sin complicaciones locales ni falla orgánica, moderada con complicaciones locales o falla orgánica (pulmonar, renal, cardiovascular, etc.) que no dura más de 48 hrs y la severa en aquella que la falla orgánica multiple persiste por más de 48hrs. ${ }^{1}$

Son complicaciones locales: áreas de parénquima pancreático no viable de más de $3 \mathrm{~cm}$, más del $30 \%$ del total del parénquima pancreático y/o colecciones pancreáticas y peripancreáticas. 3,15

Falla orgánica: como un puntaje de 2 o más en la escala modificada de Marshall. El score modificado de Marshall es la clasificación usada por el consenso de Atlanta para valorara la falla orgánica. $^{3,6}$

BISAP otro score de severidad para la pancreatitis aguda, un puntaje de 2 nos habla de pancreatitis severa, si se mantiene por más de 48 hrs. ${ }^{16}$

También se ha utilizado la proteína $C$ reactiva (PCR) con un corte de $150 \mathrm{mg} / \mathrm{dL}$ o más en las primeras 72 hrs sugestivo de pancreatitis aguda severa y es predictivo de mal pronóstico. La sensibilidad es de $85 \%$ durante las primeras $72 \mathrm{hrs}$ de empezados los síntomas. ${ }^{12,17,18}$

La escala de Ranson (1974) es un sistema de clasificación pronóstica basado en una puntuación de 11 variables, durante las primeras 48 horas de evolución La presencia de más de 6 signos de Ranson pronostica alta incidencia de complicaciones como necrosis. con sensibilidad y especificidad (70-85\%), pero su valor predictivo positivo no pasa del $50-60 \%$.

Otro sistema de clasificación pronóstica el APACHE II (Acute Physiology And Chronic Health 
Evaluation), asigna puntos a 12 variables fisiológicas, y genera una puntuación total, en el momento del ingreso y a las 48 horas.

Par efectos pronósticos, la clasificación basada en la TC es la de Balthazar. Siguiendo esta clasificación, los niveles $D$ y E se correlacionan con alta morbimortalidad. ${ }^{9}$

\section{Tratamiento}

Todos los pacientes con un cuadro de PA biliar deben ser ingresados a cargo del equipo de Cirugía General, el resto de etiologías que condicione este cuadro deberá ser visto por el equipo de gastroenterología médica. ${ }^{19}$

Dentro del tratamiento de la PA la terapia hídrica es el punto inicial más importante, su función es aumentar el retorno venoso y evitar la hipoperfusión de los tejidos causada por la pérdida al tercer espacio, favoreciendo la necrosis pancreática, en caso de no ser suficiente se utilizarán agentes vasoactivos. . $^{720,21}$

Raffaele Pezzilli et. al. ${ }^{22}$ recomiendan que dentro de las primeras 24 horas después de la admisión la resucitación hídrica debe ser a $2 \mathrm{ml} / \mathrm{kg} / \mathrm{h}$, con un bolo inicial de $20 \mathrm{ml} / \mathrm{kg}$ dentro de 30-45 minutos. La solución ideal es el Ringer Lactato, por su semejanza a la concentración sanguínea.

La American College of Gastroenterology (ACG) ha recomendado $250-500 \mathrm{ml}$ por hora dentro de las primeras 12-24 hrs con re evaluaciones cada 6 hrs, hasta obtener un descenso del nitrógeno ureico en sangre, y mantener un índice urinario de
$0.5 \mathrm{ml} / \mathrm{kg} / \mathrm{hr}$. La meta es una infusión de 2.5-4 litros en $24 \mathrm{hrs}$, basado en la edad del paciente. Un análisis retrospectivo demostró que el uso de más de 4 litros en las primeras 24 hs tuvieron más complicaciones respiratorias, asociándose a mayor mortalidad. ${ }^{7,23}$

El aumento de hematócrito y/o urea (o nitrógeno ureico en sangre) son marcadores indirectos de disminución del volumen intravascular y se asocia a mayor incidencia de necrosis pancreática y falla orgánica. ${ }^{21}$

El uso de antibióticos de manera profiláctica no está recomendado en cualquier caso de PA, este debe ser indicado únicamente en pacientes con necrosis infectada confirmada por aspiración con aguja fina o al confirmar la presencia de gas dentro de una colección por medio de una TC. ${ }^{12}$ La terapia antimicrobiana debe ser de acuerdo al resultado del cultivo o bien dirigida a las bacterias más comunes (Escherichia coli, Bacteroides species, Enterobacter species, Klebsiella species y Streptococcus faecalis, en menor de los casos Staphylococcus epidermidis y Staphylococcus aureus). ${ }^{12}$

Se ha demostrado que el uso de antibióticos puede diferir la intervención quirúrgica de una necrosis infectada hasta por 4 semanas. ${ }^{24}$

La necrosis pancreática infectada es la causa más común de muerte en la fase tardía de la PA, con hasta un $70 \%$ de mortalidad. ${ }^{7}$ Se ha reportado aumento de la morbimortalidad cuando se asocian infecciones micóticas, siendo las más comúnes especies de Candida. ${ }^{25}$ 
Parte fundamental del tratamiento es la alimentación, si la vía enteral es tolerada, se prefiere sobre la parenteral. En PA leve se debe iniciar la dieta enteral dentro de la primera semana de hospitalización. En PA severa la dieta enteral puede iniciarse dentro de las primeras $48 \mathrm{hrs}$ a $72 \mathrm{hrs}$ de hospitalización ${ }^{7,12}$ y debe ser determinado usando indicadores como el dolor abdominal y el valor de la lipasa sérica. ${ }^{11}$

En una revisión sistemática por Vaughn et al. ${ }^{26}$ la alimentación temprana fue bien tolerada, reduciendo el tiempo de hospitalización en pancreatitis leve. El uso de suplementos con glutamina no ha mostrado mejoría en la PA. ${ }^{27}$

Las complicaciones (necrosis pancreática o falla orgánica múltiple) no mostraron diferencia entre el inicio temprano o tardío de la dieta en pacientes con pancreatitis severa. Si el paciente no tolera la vía oral, por dolor, nausea, vómito o ileo se debe considerar la alimentación por sonda después de 3 a 5 dias. ${ }^{12,16}$ La sonda nasogástrica y nasoyeyunal para alimentación enteral se ha demostrado ser un método eficaz y seguro, sin diferencia entre ellas. ${ }^{26,28,29}$

La nutrición parenteral se utiliza en caso que la vía enteral no sea posible usarse o cuando por vía enteral no se completan los requerimentos necesarios, sin embargo, la vía parenteral por tiempo prolongado conlleva numerosos efectos adversos como atrofia y aumento de la permeabilidad de la mucosa intestinal. Además, la falta de peristalsis produce hipomotilidad intestinal, causando cambios en la microflora intestinal, por el contrario, la alimentación enteral facilita la motilidad intestinal debido a la hiperosmolaridad de los nutrientes. ${ }^{7}$ Como parte del tratamiento se encuentran los procedimientos invasivos, como la colangio pancreatografía retrógrada endoscópica (CPRE) y drenaje percutáneo para derivar la vía biliar, debiendo ser considerados en casos de PA severa biliar con colangitis (urgente <24hrs). ${ }^{19}$ La administración de indometacina rectal disminuyó significativamente las tasas de pancreatitis post CPRE, asi como la tasa de conversión, de PA moderada a severa. ${ }^{23,30}$

En caso de ser necesaria la intervención quirúrgica ésta, se prefiere posponer de 4 a 6 semanas de inicio de la enfermedad. ${ }^{31}$ Debido a la alta tasa de mortalidad de la cirugía en las etapas iniciales de la pancreatitis, el tratamiento quirúrgico de urgencia se reserva para situaciones que ponen en peligro la vida como la hemorragia no controlada por angiografía (sangrado arterial por pseudo aneurisma de la arteria gástrica izquierda, gastroduodenal o esplénica), perforación de víscera hueca y síndrome compartimental abdominal (presión intrabdominal mayor a $20 \mathrm{mmHg}$ asociada a datos de falla orgánica). ${ }^{3}$

La indicación para intervención quirúrgica es la sospecha o documentación de necrosis infectada o bien, en auscencia de infección, falla orgánica múltiple (FOM) persistente por varias semanas con una colección amurallada y persistencia del dolor o el ileo. ${ }^{32}$

Entre las opciones quirúrgicas se encuentran la necrosectomía percutánea, endoscópica, abierta 
u otras formas de necrosectomía minimamemnte invasivas.

La necrosectomía abierta se asocia con una alta morbilidad (34-95\%) y mortalidad (6-25\%). En el postoperatorio inmediato se puede presentar efectos adversos como FOM, perforación de viscera hueca, hemorragia e infección de la herida que requiera nuevas reintervenciones. ${ }^{33}$

El drenaje percutáneo guiado por imagen ha sido usado como terapia primaria o en combinación con otras técnicas, ${ }^{34}$ el abordaje puede ser transperitoneal o retroperitoneal, prefiriéndose este último ya que evita contaminación de la cavidad abdominal o fugas, y facilita la terapia step-up (considerándose este abordaje el primer paso de dicha terapia), se prefieren catéteres con un diámentro 12 a 30 fr. Van Baal et $\mathrm{al}^{33}$ en 2011 mostraron que el drenaje percutáneo como tratamiento primario para necrosis pancreática fue exitosos en $55.7 \%$ de los pacientes con una mortalidad de $15.4 \%$ en casos de necrosis infectada. Se reportaron algunos efectos adversos como fístulas en un $27 \%$.

En la necrosis pancreática la aspiración por aguja fina no esta indicado de manera rutinaria ya que mediante la clínica e imagen son predictores de necrosis infectada en la mayoría de los casos. ${ }^{19}$ Dentro de los abordajes de mínima invasión, el estudio multicéntrico aleatorizado TENSION, ${ }^{35}$ realiza una endoscopía intraluminal, con drenaje o necrosectomía a pacientes con pancreatitis necrotizante infectada y lo compara con el abordaje quirúrgico mínimante invasivo, concluyendo una disminución en las complicaciones y en la estancia intrahospitalaria a favor del abordaje endoscópico. Dentro de abordaje el drenaje percutáneo se realiza con una sonda 14 a 20 fr guíado por TC; en caso de necrosectomía transluminal endoscópica, esta se realiza mediante una cistogastrostomía (18mm), y por medio de un gastroscopio se realiza la necrosectomía endoscópica directa, dejándose un drenaje $7 \mathrm{Fr}$ nasoquistico.

El estudio de PENGUIN ${ }^{36}$ ha demostrado reducir las complicaciones en pacientes con necrosectomia endoscópica comparado con la cirugía abierta. Demostrando que el drenaje por medio de cistogastrostomía más desbridamiento seguido de peróxido de hidrógeno reduce las intervenciones comparados con otras técnicas quirúrgicas (el peróxido de hidrógeno es un químico desbridante que se remueve con la necrosectomía, induciendo la formación de fibrosis en la cavidad).

El estudio PENGUIN mostró que al aplicar TENSION (necrosectomía endoscópica transluminal), el síndrome de respuesta inflamatoria sistémica (SIRS) disminuye: al disminuir la concentración de IL6 en la sangre, en comparación con la cirugía mínimamente invasiva. ${ }^{24}$

Video-assisted retroperitoneal debridement VARD, es una técnica quirúrgica de mínima invasión y se considera el último paso dentro del tratamiento step-up. Utilizando el abordaje descrito en el estudio PANTER se coloca paciente en decúbito lateral izquierdo elevado a $30-40^{\circ}$, se realiza una incisión de $5 \mathrm{~cm}$ subcostal izquierda en 
línea axilar media por donde se diseca hasta localizar el espacio peripancreático, bajo visión directa, se insufla cavidad a $10 \mathrm{l} / \mathrm{m}$, procediendo al lavado de esta región para seguir con la necrosectomía con pinzas de anillos y laparoscópicas y por último se colocan drenajes de 24 a $28 \mathrm{Fr}$ uno para irrigar y otro para aspirar. ${ }^{31,37,38}$

STEP-UP se ha considerado la mejor opción para el tratamiento de le necrosis pancreática infectada, se expresan “3D” por demora, drenaje y desbridación (delay, drain, and debride). Esto significa que el manejo óptimo debe incluir como primer paso el cuidado intensivo para mejorar condiciones, esperando por lo general 4 semanas para la formación de la pared de la colección, el segundo paso es el drenaje percutáneo o endoscópico y el tercer paso, si aún se requiere, es la desbridación minimamente invasiva. ${ }^{39,40}$

En 2010 New England Journal of Medicine publicó el estudio de PANTER, demostrando la disminución de las complicaciones. Mostró que no había diferencia significativa en la mortalidad (19\% necrosectomia abierta, $16 \%$ con el abordaje STEPUP), pero si en morbilidad (12\% vs $40 \%$ ) y se han demostrado menos efectos adversos, en comparación con la necrosectomía abierta. ${ }^{24,34,41}$

Además, el estudio PANTER mostró que el 35\% de los pacientes con pancreatitis necrotizante infectada logran una recuperación completa después del drenaje percutáneo solamente, sin la necesidad de desbridamiento quirúrgico. ${ }^{35}$

Las guías de la International Association of Pancreatology 2012, recomiendan drenaje percutá- neo o endoscópico como primer paso en el tratamiento de la necrosis pancreática, seguido por necrosectomía quirúrgica solo si se requiere. ${ }^{33,42}$

\section{Complicaciones}

La necrosis pancreática se encuentra en el 5-10\% de los casos de pancreatitis aguda. Existen 3 subtipos basados en áreas anatómicas de la necrosis: a) solo pancreática, b) solo peripancreática y c) ambas $(75 \%) .6,9$

Las complicaciones locales detectadas en la primera semana generalmente no requieren intervención, únicamente requieren el tratamiento de soporte.

En la nueva clasificación de revisión de estratificación de pancreatitis dentro de dos categorías basadas en imagen: Pancreatitis Intersticial Edematosa (PIE) y necrosis pancreática, siendo la más común la PIE, donde no se observa necrosis si no únicamente inflamación del páncreas. En la TC y resonancia magnética (RM) con contraste IV, las zonas con edema intersticial muestran un realce disminuido, sin embargo, las zonas de necrosis pancreática, no mostrarán realce alguno. ${ }^{6}$ La colección líquida aguda peripancreática es una complicación temprana de la PA, homogénea, única o múltiple, sin pared definida y confinada a los planos de la fascia normal del retroperitoneo. Las colecciones pueden ser estériles o infectadas, las infectadas son más comunes en las colecciones necróticas. ${ }^{9}$

El pseudoquiste pancreático es una lesión encapsulada y con una pared de tejido inflamatorio bien 
definida, con mínima necrosis o sin ella. Su maduración ocurre después de 4 semanas desde el inicio de la pancreatitis. Puede ser debida a disrupción del conducto de Wirsung. ${ }^{9}$

La colección necrótica aguda contiene cantidades variables de líquido y necrosis, asociada con una pancreatitis necrótica en las primeras 4 semanas. La necrosis puede abarcar parénquima y tejidos peripancreáticos, no tiene una cápsula definida. Puede ser estéril o infectada. ${ }^{9}$

La necrosis encapsulada es una colección madura encapsulada de tejido pancreático bien definida y completamente encapsulada, con una mortalidad de $30 \%$. $^{9,19,36}$

El tratamiento quirúrgico para cualquiera de las complicaciones, deberá reservarse a los pacientes sintomáticos y se deberá esperar un mínimo de 6 semanas. ${ }^{3}$ La cistogastrostomía asistida por ultrasonido endoscópico ha demostrado una tasa de resolución del 45-93\% y se recomiendan en la gran mayoría de las guías clínicas publicadas. ${ }^{3,43,44}$

Otras complicaciones reportadas son: absceso intraabdominal, como resultado de la infección y encapsulación de colecciones; el pseudoaneurisma, que af ecta generalmente a la arteria gastroduodenal o esplénica; la obstrucción colónica o duodenal, originada por compresión o infiltración inflamatoria local; la trombosis esplenoportal con el desarrollo de várices gástricas como mecanismo compensatorio, 3,9,12

Se ha descrito entre un $12 \%$ a $65 \%$ de los pacientes presentan insuficiencia pancreática exócrina, hasta el 30\% de los pacientes desarrollará diabetes mellitus, El inicio temprano de suplementación enzimática pancreática ha demostrado ser benéfica únicamente en $53 \%$ de los pacientes. 3,44

La colecistectomía debe realizarse durante la estancia intraospitalaria en caso de pancreatitis leve, ya que el no hacerlo se asocia a riesgo de readmisión por recurrencia de eventos. ${ }^{42}$

En caso de colecistitis aguda si la cirugía no es posible se recomienda la esfinterotomía endoscópica, siendo suficiente para prevenir cuadros futuros de pancreatitis. ${ }^{3,12}$

En un meta-análisis de Cochrane se demostró que no existe evidencia respecto a la tasa de complicaciones en pacientes sometidos a colecistectomía laparoscópica en las primeras 48 horas de inicio del cuadro de pancreatitis aguda leve durante la estancia intrahospitalaria, en caso de colecistitis aguda. ${ }^{3,12}$

La tasa de recurrencia de pancreatitis aguda es $25-50 \%$. En caso de episodios recurrentes los estudios de imagen son recomendados para evaluar la anatomía biliar y pancreática. ${ }^{7}$

\section{Conclusiones}

Con esta revisión se espera facilitar la información acerca de la pancreatitis aguda, concepto, patología, diagnóstico y tratamiento, para mejorar la atención del paciente. Tomando en cuenta que la PA es una enfermedad multifactorial que ha tomado mayor intéres por el uso de nuevas tecnologías que han facilitado su diagnóstico y tratamiento. 


\section{Referencias}

1. Komolafe O, Pereira SP, Davidson BR, Gurusamy KS. Serum C-reactive protein, procalcitonin, and lactate dehydrogenase for the diagnosis of pancreatic necrosis. Cochrane Database Syst Rev. 2017; 21;4:CD012645.

2. Ince AT, Baysal B. Pathophysiology, classification and available guidelines of acute pancreatitis. Turk J Gastroenterol. 2014;25(4):351-7

3. González J, Luque de León E, Cedillo E, Juárez M, Gonzáles D, Moreno C, et al. Guia de práctica clínica, Pancreatitis aguda. Mèxico, DF, AMCG 2014. https://amcg.org.mx/images/guiasclinicas/pancreatitis.pdf (último acceso 19 mayo 2018).

4. Chang YC. Is necrosectomy obsolete for infected necrotizing pancreatitis? Is a paradigm shift needed? World J Gastroenterol 2014;20(45):16925 -34.

5. Camacho AV, Morales MLA, Jiménez RD, Borjas FGE. Apuntes históricos sobre el tratamiento quirúrgico de la pancreatitis aguda severa. Mediciego 2013;19(2)

6. Foster BR, Jensen KK, Bakis G, Shaaban AM, Coakley FV. Revised Atlanta Classification for Acute Pancreatitis: A Pictorial Essay. Radiographics 2016;36(3):675-87.

7. Janisch NH, Gardner TB. Advances in Management of Acute Pancreatitis. Gastroenterol Clin North Am. 2016; 45(1):1-8.

8. Poropat G, Giljaca V, Hauser G, Štimac D. Enteral nutrition formulations for acute pancreatitis. Cochrane Database Syst Rev 2015;23(3):CD010605.

9. Alberca de las Parras F, Sánchez E y Carballo F. Pancreatitis aguda. Medicine.
2016;12(8):407-420.

10. Tuennemann J, Mössner J, Beer S. Acute pancreatitis: guideline-based diagnosis and treatment (german). Der Internist 2014;55(9):104556 .

11. Yokoe M, Takada T, Mayumi T, Yoshida M, Isaji $\mathrm{S}$, Wada $\mathrm{H}$, et. al. Japanese guidelines for the management of acute pancreatitis: Japanese Guidelines 2015. J Hepatobiliary Pancreat Sci 2015;22(6):405-32.

12. Greenberg JA, Hsu J, Bawazeer M, Marshall J, Friedrich JO, Nathens A, et al. Clinical practice guideline: management of acute pancreatitis. Can J Surg. 2016;59 (2):128-40.

13. Murphy KP, O'Connor OJ, Maher MM. Updated Imaging Nomenclature for acute pancreatitis. AJR Am J Roentgenol. 2014;203(5):W 464-9.

14. Baker M, Nelson R, Rosen M, Blake M, Cash B, Hindman N, et. al. Acute Pancreatitis. ACR Appropriateness Criteria. 2013:1-11.

15. Hritz I, Czakó L, Dubravcsik Z, Farkas G, Kelemen $\mathrm{D}$, Lásztity N, et al. Acute pancreatitis. Evidence-based practice guidelines, prepared by the Hungarian Pancreatic Study Group (Hungarian). Orv Hetil. 2015;156(7):244-61.

16. Chandra S, Murali A, Bansa R, Agarwal D, Holm A. The Bedside Index for Severity in Acute Pancreatitis: a systematic review of prospective studies to determine predictive performance. $J$ Community Hosp Intern Med Perspect. 2017;7(4):208-13.

17. Bereanu A, Kiss L, Porr PJ, Sava M, Kiss R. Severe Acute Pancreatitis (SAP) Review of Literature. AMT 2013;18(2):296-8.

18. Komolafe O, Pereira SP, Davidson BR, Gurusamy K. Análisis de sangre para el diagnóstico 
de necrosis pancreática (destrucción pancreática por inflamación del páncreas) Cochrane Database Syst Rev. 2017;4:CD012645.

19. Clarke M, Mohamed A. Clinical Guideline for Management of acute pancreatitis in Adults. Royal Cornwall Hospital. NHS Truts. 2017. https://doclibrary-rcht.cornwall.nhs.uk/DocumentsLibrary/RoyalCornwallHospitalsTrust/Clinical/GeneralSurgery/117. AcutePancreatitisinAdultsGuidelineforManagementof.pdf (último acceso 19 mayo 2018).

20. Hart P, Eldika S, Ramsey M, Conwell D, Shah Z. Management of Acute Pancreatitis (AP). The Ohio State University Wexner Medical Center. 2016. https://evidencebasedpractice.osumc.edu/Documents/Guidelines/AcutePancreatitis.pdf (último acceso 19 mayo 2018).

21. De-Madaria E. Fluidoterapia en la pancreatitis aguda. Gastroenterol Hepatol. 2013;36(10):63140.

22. Pezzilli R, Zerbi A, Campra D, Capurso G, Golfieri R, Arcidiacono PG, et al. Consensus Guidelines on severe acute pancreatitis. Dig Liver Dis. 2015;47(7):532:43.

23. Tenner S, Baillie J, DeWitt J, Vege SS. American College of Gastroenterology guideline: management of acute pancreatitis. Am J Gastroenterol. 2013;108(9):1400-15.

24. Šileikis A, Pečiulyté E, Misenkienė A. Klimašauskas A, Beiša V, Strupas $K$. Is minimally invasive surgical treatment justified for severe acute necrotizing pancreatitis patients with dysfunction of two or more organ systems? Videosurgery Miniinv. 2017;12(3):225-230.

25. De Waele JJ. Acute pancreatitis. Curr Opin Crit Care 2014;20(2):189-95.

26. Vaughn VM, Shuster D, Rogers MAM, et al.
Early versus delayed feeding in patients with acute pancreatitis: a systematic review. Ann Intern Med. 2017;166:883-92.

27. Jeurnik SM, Nijs MM, Prins HA, Greving JP, Siersema PD. Antioxidants as a treatment for acute pancreatitis: A meta-analysis. Pancreatology 2015;15(3): 203-8

28. Dutta AK, Goel A, Kirubakaran R, Chacko A. Nasogastric versus nasojejunal tube feeding for severe acute pancreatitis. Cochrane Database Syst Rev 2013;6:CD010582

29. Oláh A, Romics Jr L. Enteral nutrition in acute pancreatitis: A review of the current evidence. World J Gastroenterol. 2014;20(43):16123-31.

30. Thiruvengadam N, Forde K, Ma G. Rectal Indomethacin Reduces Pancreatitis in High- and Low-Risk Patients Undergoing Endoscopic Retrograde Cholangiopancreatography. Gastroenterology 2016;151(2):288-97.

31. Van Santvoort HC, Besselink, MGH, Horvath KD, Sinanan MN, Bollen TL, Ramshorst, et. al. Videoscopic assisted retroperitoneal debridement in infected necrotizing pancreatitis. HPB. 2007;9(2):156-9.

32. Logue J, Carter R. Minimally Invasive Necrosectomy Techniques in Severe Acute Pancreatitis: Role of Percutaneous Necrosectomy and VideoAssisted Retroperitoneal Debridement. Gastroenterol Res Pract. 2015;2015:693040.

33. Bugiantella W, Rondelli F, Boni M, Stella $P$, Polistena $A$, Sanguinetti $A$, et al. Necrotizing pancreatitis: A review of the interventions. Int $\mathrm{J}$ Surg. 2016;28:S163-71.

34. Gurusamy KS, Belgaumkar AP, Haswell A, Pereira SP, Davidson BR. Interventions for necrotising pancreatitis. Cochrane Database Syst Rev. 2016;4:CD011383. 
35. Van Brunschot S, Van Grinsven J, Voermans RP, Bakker OJ, Besselink MG, Boermeester MA, et. al. Transluminal endoscopic step-up approach versus minimally invasive surgical stepup approach in patients with infected necrotising pancreatitis (TENSION trial): design and rationale of a randomised controlled multicenter trial [ISRCTN09186711]. BMC Gastroenterol. 2013;13(161):1-13.

36. Othman M, Elhanafi S, Saadi M, Yu Ch, Davis B. Extended Cystogastrostomy with Hydrogen Peroxide Irrigation Facilitates Endoscopic Pancreatic Necrosectomy. Diagn Ther Endosc. 2017; 2017:7145803.

37. Szeliga J, Jackowski M. Minimally invasive procedures in severe acute pancreatitis treatment assessment of benefits and possibilities of use. Videosurgery Miniinv 2014;9(2):170-8.

38. Zhao G, Hu M, Liu R, Xu Y. Retroperitoneoscopic Anatomical Necrosectomy: A Modified Single-Stage Video-Assisted Retroperitoneal Approach for Treatment of Infected Necrotizing Pancreatitis. Surg Innov. 2014;22(4):360-365.

39. Tu Y, Jiao H, Tan X. Sun L, Zhang W. Laparotomy versus retroperitoneal laparoscopy in debridement and drainage of retroperitoneal infected necrosis in severe acute pancreatitis. Surg Endosc. 2013; 27(11):4217-23.
40. Sorrentino L, Chiara O, Mutignani M, Sammartano F, Brioschi P, Cimbanassi S. Combined totally mini-invasive approach in necrotizing pancreatitis: a case report and systematic literature review. World J Emerg Surg. 2017;12(16):1-14.

41. Van Santvoort $H$, Besselink M, Bakker O, Hofker $\mathrm{HS}$, Boermeester MA, Dejong $\mathrm{CH}$, et. al. A stepup approach or open necrosectomy for necrotizing pancreatitis. $N$ Engl J Med 2010;362(10):1491-1502.

42. Working Group IAP/APA Acute Pancreatitis Guidelines. IAP/APA evidence-based guidelines for the management of acute pancreatitis. Pancreatology. 2013.13(4 Suppl 2):e1-15.

43. Gibson SC, Robertson BF, Dickson EJ, McKay CJ, Carter CR. 'Step-port' laparoscopic cystgastrostomy for the management of organized solid predominant post-acute fluid collections after severe acute pancreatitis. HPB 2014;16(2):170-6.

44. Wu BU, Banks PA. Clinical management of patients with acute pancreatitis. Gastroenterology. 2013;144(6):1272-81. 